\title{
ON I-CONVERGENCE OF SEQUENCES IN GRADUAL NORMED LINEAR SPACES
}

\author{
Chiranjib Choudhury and Shyamal Debnath \\ Department of Mathematics, Tripura University(A Central University), \\ Suryamaninagar-799022, Agartala, India
}

\begin{abstract}
In this paper, we introduce the concepts of $\mathcal{I}$ and $\mathcal{I}^{*}$-convergence of sequences in gradual normed linear spaces. We study some basic properties and implication relations of the newly defined convergence concepts. Also, we introduce the notions of $\mathcal{I}$ and $\mathcal{I}^{*}$-Cauchy sequences in the gradual normed linear space and investigate the relations between them.

Keywords: Gradual number; gradual normed linear space; ideal; filter; ideal convergence.
\end{abstract}

\section{Introduction}

The idea of fuzzy sets [20] was first introduced by Zadeh in the year 1965 which was an extension of the classical set-theoretical concept. Nowadays, it has wide applicability in different branches of science and engineering. The term "fuzzy number" plays a crucial role in the study of fuzzy set theory. Fuzzy numbers were basically the generalization of intervals, not numbers. Even fuzzy numbers do not obey a few algebraic properties of the classical numbers. So the term "fuzzy number" is debatable to many authors due to its different behavior. The term "fuzzy intervals" is often used by many authors instead of fuzzy numbers. To overcome the confusion among the researchers, in 2008, Fortin et.al. [8] introduced the notion of gradual real numbers as elements of fuzzy intervals. Gradual real numbers are

Received January 08, 2021. accepted April 28, 2021.

Communicated by Dijana Mosić

Corresponding Author: Shyamal Debnath, Department of Mathematics, Tripura University(A Central University),Suryamaninagar-799022, Agartala, India | E-mail: shyamalnitamath@gmail.com

2010 Mathematics Subject Classification. Primary 03E72; Secondary 40A35, 40 A05

(C) 2021 by University of Niš, Serbia | Creative Commons License: CC BY-NC-ND 
mainly known by their respective assignment function which is defined in the interval $(0,1]$. So in some sense, every real number can be viewed as a gradual number with a constant assignment function. The gradual real numbers also obey all the algebraic properties of the classical real numbers and have uses in computation and optimization problems.

In 2011, Sadeqi and Azari [15] first introduced the concept of gradual normed linear space. They studied various properties of the space from both the algebraic and topological point of view. Further progress in this direction has been occurred due to Ettefagh, Azari, and Etemad (see [6],[7]) and many others. For extensive study on gradual real numbers one may refer to $([1],[5][12],[18],[21],[22])$.

On the other hand in 2001, the idea of ideal convergence was first introduced by Kostyrko et al. [11] mainly as an extension of statistical convergence. They also showed that ideal convergence was also a generalized form of some other known convergence concepts. Later on, several investigations in this direction have been occurred due to Debnath and Rakshit [2], Demirci [3], Gogola et al. [9], Mursaleen and Mohiuddine [13], Savas and Das[17] and many others. For an extensive view of this article, we refer to $[4,10,14,16,19]$.

Research on the convergence of sequences in gradual normed linear spaces has not yet gained much ground and it is still in its infant stage. The research carried out so far shows a strong analogy in the behavior of convergence of sequences in gradual normed linear spaces (for details one may refer to $[6],[7],[15]$ ).

Recently, the convergence of sequences in gradual normed linear spaces was introduced by Ettefagh et. al. [7]. Also, they have investigated some properties from the topological point of view [6]. Therefore, the study of ideal convergence of sequences in gradual normed linear spaces is very natural.

\section{Definitions and Preliminaries}

Definition 2.1. [8] A gradual real number $\tilde{r}$ is defined by an assignment function $A_{\tilde{r}}:(0,1] \rightarrow \mathbb{R}$. The set of all gradual real numbers is denoted by $G(\mathbb{R})$. A gradual number is said to be non-negative if for every $\xi \in(0,1], A_{\tilde{r}}(\xi) \geq 0$. The set of all non-negative gradual real numbers is denoted by $G^{*}(\mathbb{R})$.

In [8], the gradual operations between the elements of $G(\mathbb{R})$ was defined as follows:

Definition 2.2. Let $*$ be any operation in $\mathbb{R}$ and suppose $\tilde{r}_{1}, \tilde{r}_{2} \in G(\mathbb{R})$ with assignment functions $A_{\tilde{r}_{1}}$ and $A_{\tilde{r}_{2}}$ respectively. Then $\tilde{r}_{1} * \tilde{r}_{2} \in G(\mathbb{R})$ is defined with the assignment function $A_{\tilde{r}_{1} * \tilde{r}_{2}}$ given by $A_{\tilde{r}_{1} * \tilde{r}_{2}}(\xi)=A_{\tilde{r}_{1}}(\xi) * A_{\tilde{r}_{2}}(\xi), \forall \xi \in(0,1]$. Then the gradual addition $\tilde{r}_{1}+\tilde{r}_{2}$ and the gradual scalar multiplication $c \tilde{r}(c \in \mathbb{R})$ are defined by

$$
A_{\tilde{r}_{1}+\tilde{r}_{2}}(\xi)=A_{\tilde{r}_{1}}(\xi)+A_{\tilde{r}_{2}}(\xi) \quad \text { and } \quad A_{c \tilde{r}}(\xi)=c A_{\tilde{r}}(\xi), \quad \forall \xi \in(0,1] .
$$


For any real number $p \in \mathbb{R}$, the constant gradual real number $\tilde{p}$ is defined by the constant assignment function $A_{\tilde{p}}(\xi)=p$ for any $\xi \in(0,1]$. In particular, $\tilde{0}$ and $\tilde{1}$ are the constant gradual numbers defined by $A_{\tilde{0}}(\xi)=0$ and $A_{\tilde{1}}(\xi)=1$ respectively. One can easily verify that $G(\mathbb{R})$ with the gradual addition and multiplication forms a real vector space [8].

Definition 2.3. [15] Let $X$ be a real vector space. The function $\|\cdot\|_{G}: X \rightarrow$ $G^{*}(\mathbb{R})$ is said to be a gradual norm on $X$, if for every $\xi \in(0,1]$, following three conditions are true for any $x, y \in X$

$\left(G_{1}\right) A_{\|x\|_{G}}(\xi)=A_{\tilde{0}}(\xi)$ iff $x=0$;

$\left(G_{2}\right) A_{\|\lambda x\|_{G}}(\xi)=|\lambda| A_{\|x\|_{G}}(\xi)$ for any $\lambda \in \mathbb{R}$;

$\left(G_{3}\right) A_{\|x+y\|_{G}}(\xi) \leq A_{\|x\|_{G}}(\xi)+A_{\|y\|_{G}}(\xi)$.

The pair $\left(X,\|\cdot\|_{G}\right)$ is called a gradual normed linear space (GNLS).

Example 2.1. [15] Let $X=\mathbb{R}^{n}$ and for $x=\left(x_{1}, x_{2}, \ldots, x_{n}\right) \in \mathbb{R}^{n}, \xi \in(0,1]$, define $\|\cdot\|_{G}$ by $A_{\|x\|_{G}}(\xi)=e^{\xi} \sum_{i=1}^{n}\left|x_{i}\right|$. Then $\|\cdot\|_{G}$ is a gradual norm on $\mathbb{R}^{n}$ and $\left(\mathbb{R}^{n},\|\cdot\|_{G}\right)$ is a GNLS.

Definition 2.4. [15] Let $\left(x_{k}\right)$ be a sequence in the $\operatorname{GNLS}\left(X,\|\cdot\|_{G}\right)$. Then $\left(x_{k}\right)$ is said to be gradual convergent to $x \in X$, if for every $\xi \in(0,1]$ and $\varepsilon>0$, there exists $N\left(=N_{\varepsilon}(\xi)\right) \in \mathbb{N}$ such that $A_{\left\|x_{k}-x\right\|_{G}}(\xi)<\varepsilon, \forall n \geq N$.

Definition 2.5. [15] Let $\left(x_{k}\right)$ be a sequence in the $\operatorname{GNLS}\left(X,\|\cdot\|_{G}\right)$. Then $\left(x_{k}\right)$ is said to be gradual Cauchy, if for every $\xi \in(0,1]$ and $\varepsilon>0$, there exists $N(=$ $\left.N_{\varepsilon}(\xi)\right) \in \mathbb{N}$ such that $A_{\left\|x_{k}-x_{j}\right\|_{G}}(\xi)<\varepsilon, \forall k, j \geq N$.

Theorem 2.1. ([15], Theorem 3.6) Let $\left(X,\|\cdot\|_{G}\right)$ be a GNLS, then every gradual convergent sequence in $X$ is also a gradual Cauchy sequence.

Definition 2.6. [11] Let $X$ is a non-empty set. A family of subsets $\mathcal{I} \subset P(X)$ is called an ideal on $X$ if and only if

(i) $\varnothing \in \mathcal{I}$;

(ii) for each $A, B \in \mathcal{I}$ implies $A \cup B \in \mathcal{I}$;

(iii) for each $A \in \mathcal{I}$ and $B \subset A$ implies $B \in \mathcal{I}$.

Some standard examples of ideal are given below:

(i) The set $\mathcal{I}_{f}$ of all finite subsets of $\mathbb{N}$ is an admissible ideal in $\mathbb{N}$. Here $\mathbb{N}$ denotes the set of all natural numbers.

(ii) The set $\mathcal{I}_{d}$ of all subsets of natural numbers having natural density 0 is an admissible ideal in $\mathbb{N}$.

(iii) The set $\mathcal{I}_{c}=\left\{A \subseteq \mathbb{N}: \sum_{a \in A} a^{-1}<\infty\right\}$ is an admissible ideal in $\mathbb{N}$.

(iv) Suppose $\mathbb{N}=\bigcup_{p=1}^{\infty} D_{p}$ be a decomposition of $\mathbb{N}$ (for $i \neq j, D_{i} \cap D_{j}=\varnothing$ ). Then the set $\mathcal{I}$ of all subsets of $\mathbb{N}$ which intersects finitely many $D_{p}$ 's forms an ideal in $\mathbb{N}$.

More important examples can be found in [9] and [10]. 
Definition 2.7. [11] Let $X$ be a non-empty set. A family of subsets $\mathcal{F} \subset P(X)$ is called a filter on $X$ if and only if

(i) $\varnothing \notin \mathcal{F}$;

(ii) for each $A, B \in \mathcal{F}$ implies $A \cap B \in \mathcal{F}$;

(iii) for each $A \in \mathcal{F}$ and $B \supset A$ implies $B \in \mathcal{F}$.

An ideal $\mathcal{I}$ is called non-trivial if $\mathcal{I} \neq \varnothing$ and $X \notin \mathcal{I}$. The filter $\mathcal{F}=\mathcal{F}(\mathcal{I})=$ $\{X-A: A \in \mathcal{I}\}$ is called the filter associated with the ideal $\mathcal{I}$. A non-trivial ideal $\mathcal{I} \subset P(X)$ is called an admissible ideal in $X$ if and only if $\mathcal{I} \supset\{\{x\}: x \in X\}$.

Definition 2.8. [11] Let $\mathcal{I} \subset P(\mathbb{N})$ be a non-trivial ideal on $\mathbb{N}$. A real sequence $\left(x_{k}\right)$ is said to be $\mathcal{I}$-convergent to $l$ if for each $\varepsilon>0$, the set $C(\varepsilon)=$ $\left\{k \in \mathbb{N}:\left|x_{k}-l\right| \geq \varepsilon\right\}$ belongs to $\mathcal{I}$. $l$ is called the $\mathcal{I}$-limit of the sequence $\left(x_{k}\right)$ and is written as $\mathcal{I}$-lim lim $_{k \rightarrow \infty} x_{k}=l$.

Definition 2.9. [11] Let $\mathcal{I}$ be an admissible ideal in $\mathbb{N}$. A sequence $x=\left(x_{k}\right)$ is said to be $\mathcal{I}^{*}$-convergent to $l$, if there exists a set $M=\left\{m_{1}<m_{2}<\ldots<m_{k}<\ldots\right\}$ in the associated filter $\mathcal{F}(\mathcal{I})$ such that $\lim _{k \in M} x_{k}=l$.

Definition 2.10. [14] A sequence $\left(x_{k}\right)$ of real numbers is said to be $\mathcal{I}$-Cauchy, if for every $\varepsilon>0$, there exists a $N \in \mathbb{N}$ such that $\left\{k \in \mathbb{N}:\left|x_{k}-x_{N}\right| \geq \varepsilon\right\} \in \mathcal{I}$.

Definition 2.11. [14] A sequence $\left(x_{k}\right)$ of real numbers is said to be $\mathcal{I}^{*}$-Cauchy, if there exists a set $M=\left\{m_{1}<m_{2}<\ldots<m_{i}<\ldots\right\} \subset \mathbb{N}, M \in \mathcal{F}(\mathcal{I})$ such that the subsequence $\left(x_{m_{k}}\right)$ is a Cauchy sequence i.e. $\lim _{i, j \rightarrow \infty}\left|x_{m_{i}}-x_{m_{j}}\right|=0$.

Definition 2.12. [11] An admissible ideal $\mathcal{I}$ is said to satisfy the condition AP, if for every countable family of mutually disjoint sets $\left\{C_{n}\right\}_{n \in \mathbb{N}}$ from $\mathcal{I}$, there exists a countable family of sets $\left\{B_{n}\right\}_{n \in \mathbb{N}}$ such that the symmetric difference $C_{j} \triangle B_{j}$ is finite for every $j \in \mathbb{N}$ and $\bigcup_{j=1}^{\infty} B_{j} \in \mathcal{I}$.

Throughout the article $\mathcal{I}$ will denote the non-trivial admissible ideal of $\mathbb{N}$.

\section{Main Results}

Definition 3.1. Let $\left(x_{k}\right)$ be a sequence in the GNLS $\left(X,\|\cdot\|_{G}\right)$. Then $\left(x_{k}\right)$ is said to be gradually $\mathcal{I}$-convergent to $x \in X$ if for every $\xi \in(0,1]$ and $\varepsilon>0$, the set $C(\xi, \varepsilon)=\left\{k \in \mathbb{N}: A_{\left\|x_{k}-x\right\|_{G}}(\xi) \geq \varepsilon\right\} \in \mathcal{I}$. Symbolically we write, $x_{k} \stackrel{\mathcal{I}-\|\cdot\|_{G}}{\longrightarrow} x$.

Example 3.1. Let $X=\mathbb{R}^{n}$ and $\|\cdot\|_{G}$ be the norm defined in Example 2.1. Consider the ideal $\mathcal{I}$ consisting of all subsets of $\mathbb{N}$ which intersects finitely many $D_{p}$ 's where $D_{p}=$ $\left\{2^{p-1}(2 s-1): s \in \mathbb{N}\right\}, p \in \mathbb{N}$ is the decomposition of $\mathbb{N}$ into disjoint subsets i.e $\mathbb{N}=$ 
$\bigcup_{p=1}^{\infty} D_{p}$ and $D_{i} \cap D_{j}=\varnothing$ for $i \neq j$. Consider the sequence $\left(x_{k}\right)$ in $\mathbb{R}^{n}$ defined by $x_{k}=$ $\left(0,0, \ldots, 0, \frac{1}{p}\right)$, if $k \in D_{p}$. Then $x_{k} \stackrel{\mathcal{I}-\|\cdot\|_{G}}{\longrightarrow} \mathbf{0}$ where $\mathbf{0}$ denotes the vector $(0,0, \ldots, 0) \in \mathbb{R}^{n}$.

Justification. It is obvious that $A_{\left\|x_{k}-\mathbf{0}\right\|_{G}}(\xi)=\frac{1}{p} e^{\xi}$ for $k \in D_{p}$. Let $\varepsilon>0$ be given. Then by Archimedean property, there exists $m \in \mathbb{N}$ such that $\frac{1}{m} e^{\xi}<\varepsilon$ and consequently, the following inclusion is true,

$$
\left\{k \in \mathbb{N}: A_{\left\|x_{k}-\mathbf{0}\right\|_{G}}(\xi) \geq \varepsilon\right\} \subseteq\left\{k \in \mathbb{N}: A_{\left\|x_{k}-\mathbf{0}\right\|_{G}}(\xi) \geq \frac{1}{m} e^{\xi}\right\}
$$

and as $A_{\left\|x_{k}-\mathbf{0}\right\|_{G}}(\xi)=\frac{1}{p} e^{\xi}$ for $k \in D_{p}$, we have

$$
\left\{k \in \mathbb{N}: A_{\left\|x_{k}-\mathbf{0}\right\|_{G}}(\xi) \geq \frac{1}{m} e^{\xi}\right\}=\bigcup_{p=1}^{m} D_{p} \in \mathcal{I} .
$$

From (3.1) and (3.2), we obtain $\left\{k \in \mathbb{N}: A_{\left\|x_{k}-\mathbf{0}\right\|_{G}}(\xi) \geq \varepsilon\right\} \in \mathcal{I}$. Hence $x_{k} \stackrel{\mathcal{I}-\|\cdot\|_{G}}{\longrightarrow}$ 0.

Theorem 3.1. Let $\left(X,\|\cdot\|_{G}\right)$ be a GNLS. If a sequence $\left(x_{k}\right)$ is gradual convergent to $x \in X$, then $\left(x_{k}\right)$ is gradually $\mathcal{I}$-convergent to $x \in X$.

Proof. Proof follows directly from the fact that $\mathcal{I}_{f} \subset \mathcal{I}$.

But the converse of Theorem 3.1 is not true. Example 3.2 illustrates the fact.

Example 3.2. Let $X=\mathbb{R}^{n}$ and $\|\cdot\|_{G}$ be the norm defined in Example 2.1. Consider the sequence $\left(x_{k}\right)$ in $\mathbb{R}^{n}$ defined as

$$
x_{k}= \begin{cases}(0,0, \ldots, 0, n) & \text { if } k=p^{2}, p \in \mathbb{N} \\ (0,0, \ldots .0,0) & \text { otherwise } .\end{cases}
$$

Let $\mathbf{0}$ denotes the vector $(0,0, \ldots .0,0) \in \mathbb{R}^{n}$. Then for any $\varepsilon>0$ and $\xi \in(0,1],\{k \in \mathbb{N}$ : $\left.A_{\left\|x_{k}-\mathbf{0}\right\|_{G}}(\xi) \geq \varepsilon\right\} \subseteq\{1,4,9, ..\} \in \mathcal{I}_{d}$. Hence $x_{k} \stackrel{\mathcal{I}_{d}-\|\cdot\|_{G}}{\longrightarrow} \mathbf{0}$ in $\mathbb{R}^{n}$.

Theorem 3.2. Let $\left(x_{k}\right)$ be any sequence in the $\operatorname{GNLS}\left(X,\|\cdot\|_{G}\right)$ such that $x_{k} \stackrel{\mathcal{I}-\|\cdot\|_{G}}{\longrightarrow}$ $x$ in $X$. Then $x$ is uniquely determined.

Proof. If possible suppose $x_{k} \stackrel{\mathcal{I}-\|\cdot\|_{G}}{\longrightarrow} x$ and $x_{k} \stackrel{\mathcal{I}-\|\cdot\|_{G}}{\longrightarrow} y$ for some $x \neq y$ in $X$. Let $\varepsilon>0$ be arbitrary. Then, for any $\varepsilon>0$ and $\xi \in(0,1]$, we have, $B_{1}(\xi, \varepsilon), B_{2}(\xi, \varepsilon) \in$ $\mathcal{F}(\mathcal{I})$ where $B_{1}(\xi, \varepsilon)=\left\{k \in \mathbb{N}: A_{\left\|x_{k}-x\right\|_{G}}(\xi)<\varepsilon\right\}$ and $B_{2}(\xi, \varepsilon)=\{k \in \mathbb{N}:$ $\left.A_{\left\|x_{k}-y\right\|_{G}}(\xi)<\varepsilon\right\}$. Clearly $B_{1}(\xi, \varepsilon) \cap B_{2}(\xi, \varepsilon) \in \mathcal{F}(\mathcal{I})$ and is non-empty. Choose $m \in B_{1}(\xi, \varepsilon) \cap B_{2}(\xi, \varepsilon)$, then $A_{\left\|x_{m}-x\right\|_{G}}(\xi)<\varepsilon$ and $A_{\left\|x_{m}-y\right\|_{G}}(\xi)<\varepsilon$. Hence $A_{\|x-y\|_{G}}(\xi) \leq A_{\left\|x_{m}-x\right\|_{G}}(\xi)+A_{\left\|x_{m}-y\right\|_{G}}(\xi)<\varepsilon+\varepsilon=2 \varepsilon$. Since $\varepsilon$ is arbitrary, so $A_{\|x-y\|_{G}}(\xi)=A_{\tilde{0}}(\xi)$, which gives $x=y$. 
Theorem 3.3. Let $\left(x_{k}\right)$ and $\left(y_{k}\right)$ be two sequences in the GNLS $\left(X,\|\cdot\|_{G}\right)$ such that $x_{k} \stackrel{\mathcal{I}-\|\cdot\|_{G}}{\longrightarrow} x$ and $y_{k} \stackrel{\mathcal{I}-\|\cdot\|_{G}}{\longrightarrow} y$. Then

(i) $x_{k}+y_{k} \stackrel{\mathcal{I}-\|\cdot\|_{G}}{\longrightarrow} x+y$ and (ii) $c x_{k} \stackrel{\mathcal{I}-\|\cdot\|_{G}}{\longrightarrow} c x$.

Proof. (i) Suppose $x_{k} \stackrel{\mathcal{I}-\|\cdot\|_{G}}{\longrightarrow} x$ and $y_{k} \stackrel{\mathcal{I}-\|\cdot\|_{G}}{\longrightarrow} y$. Then, for given $\varepsilon>0$, we have, $C_{1}(\xi, \varepsilon), C_{2}(\xi, \varepsilon) \in \mathcal{I}$ where $C_{1}(\xi, \varepsilon)=\left\{k \in \mathbb{N}: A_{\left\|x_{k}-x\right\|_{G}}(\xi) \geq \frac{\varepsilon}{2}\right\}$ and $C_{2}(\xi, \varepsilon)=\left\{k \in \mathbb{N}: A_{\left\|y_{k}-y\right\|_{G}}(\xi) \geq \frac{\varepsilon}{2}\right\}$. Now as the inclusion $\left(\mathbb{N} \backslash C_{1}(\xi, \varepsilon)\right) \cap(\mathbb{N} \backslash$ $\left.C_{2}(\xi, \varepsilon)\right) \subseteq\left\{k \in \mathbb{N}: A_{\left\|x_{k}+y_{k}-x-y\right\|_{G}}(\xi)<\varepsilon\right\}$ holds, so we must have

$$
\left\{k \in \mathbb{N}: A_{\left\|x_{k}+y_{k}-x-y\right\|_{G}}(\xi) \geq \varepsilon\right\} \subseteq C_{1}(\xi, \varepsilon) \cup C_{2}(\xi, \varepsilon) \in \mathcal{I}
$$

and consequently, $x_{k}+y_{k} \stackrel{\mathcal{I}-\|\cdot\|_{G}}{\longrightarrow} x+y$.

(ii) If $c=0$, then there is nothing to prove. So let us assume $c \neq 0$. Then since $x_{k} \stackrel{\mathcal{I}-\|\cdot\|_{G}}{\longrightarrow} x$, we have for given $\varepsilon>0, C_{1}(\xi, \varepsilon) \in \mathcal{I}$ where $C_{1}(\xi, \varepsilon)=\{k \in \mathbb{N}$ : $\left.A_{\left\|x_{k}-x\right\|_{G}}(\xi) \geq \frac{\varepsilon}{|c|}\right\}$. Now since $A_{\left\|c x_{k}-c x\right\|_{G}}(\xi)=|c| A_{\left\|x_{k}-x\right\|_{G}}(\xi)$ holds for any $c \in$ $\mathbb{R}$, we must have $C_{2}(\xi, \varepsilon) \subseteq C_{1}(\xi, \varepsilon)$ where $C_{2}(\xi, \varepsilon)=\left\{k \in \mathbb{N}: A_{\left\|c x_{k}-c x\right\|_{G}}(\xi) \geq \varepsilon\right\}$, which as a consequence implies $C_{2}(\xi, \varepsilon) \in \mathcal{I}$. This completes the proof.

Theorem 3.4. Let $\left(x_{k}\right)$ be any sequence in the $G N L S\left(X,\|\cdot\|_{G}\right)$. If every subsequence of $\left(x_{k}\right)$ is gradually $\mathcal{I}$-convergent to $x$, then $\left(x_{k}\right)$ is also gradually $\mathcal{I}$-convergent to $x$.

Proof. If possible suppose $\left(x_{k}\right)$ is not gradually $\mathcal{I}$-convergent to $x$. Then there exists some $\varepsilon>0$ and $\xi \in(0,1]$ such that $C(\xi, \varepsilon) \notin \mathcal{I}$, where $C(\xi, \varepsilon)=\{k \in \mathbb{N}$ : $\left.A_{\left\|x_{k}-x\right\|_{G}}(\xi) \geq \varepsilon\right\}$. So $C(\xi, \varepsilon)$ must be an infinite set. Let $C(\xi, \varepsilon)=\left\{k_{1}<k_{2}<\right.$ $\left.\ldots<k_{j}<\ldots\right\}$. Now define a sequence $\left(y_{j}\right)$ as $y_{j}=x_{k_{j}}$ for $j \in \mathbb{N}$. Then $\left(y_{j}\right)$ is a subsequence of $\left(x_{k}\right)$ which is not gradually $\mathcal{I}$-convergent to $x$, a contradiction.

Remark 3.1. Converse of the above theorem is not true.

Proof. Easy so omitted. One can verify it by considering Example 3.2 also.

Theorem 3.5. Let $\left(x_{k}\right)$ and $\left(y_{k}\right)$ be two sequences in the GNLS $\left(X,\|\cdot\|_{G}\right)$ such that $\left(y_{k}\right)$ is gradual convergent and $\left\{k \in \mathbb{N}: x_{k} \neq y_{k}\right\} \in \mathcal{I}$. Then $\left(x_{k}\right)$ is gradually $\mathcal{I}$-convergent.

Proof. Suppose $\left\{k \in \mathbb{N}: x_{k} \neq y_{k}\right\} \in \mathcal{I}$ holds and $y_{k} \stackrel{\|\cdot\|_{G}}{\longrightarrow} y$. Then by definition for every $\varepsilon>0$ and $\xi \in(0,1],\left\{k \in \mathbb{N}: A_{\left\|y_{k}-y\right\|_{G}}(\xi) \geq \varepsilon\right\}$ is a finite set and therefore

$$
\left\{k \in \mathbb{N}: A_{\left\|y_{k}-y\right\|_{G}}(\xi) \geq \varepsilon\right\} \in \mathcal{I} .
$$

Now since the inclusion

$$
\left\{k \in \mathbb{N}: A_{\left\|x_{k}-y\right\|_{G}}(\xi) \geq \varepsilon\right\} \subseteq\left\{k \in \mathbb{N}: A_{\left\|y_{k}-y\right\|_{G}}(\xi) \geq \varepsilon\right\} \cap\left\{k \in \mathbb{N}: x_{k} \neq y_{k}\right\}
$$


holds, so using Equation (3.3) and the hypothesis we get,

$$
\left\{k \in \mathbb{N}: A_{\left\|x_{k}-y\right\|_{G}}(\xi) \geq \varepsilon\right\} \in \mathcal{I} .
$$

Hence $x_{k} \stackrel{\mathcal{I}-\|\cdot\|_{G}}{\longrightarrow} y$ and the proof is complete.

Definition 3.2. Let $\mathcal{I}$ be an admissible ideal in $\mathbb{N}$ and $\left(x_{k}\right)$ be a sequence in the $\operatorname{GNLS}\left(X,\|\cdot\|_{G}\right)$. Then $\left(x_{k}\right)$ is said to be gradually $\mathcal{I}^{*}$-convergent to $x \in X$ if there exists a set $M=\left\{m_{1}<m_{2}<\ldots<m_{k}<\ldots\right\} \in \mathcal{F}(\mathcal{I})$ such that the subsequence $\left(x_{m_{k}}\right)$ is gradual convergent to $x$. Symbolically we write, $x_{k} \stackrel{\mathcal{I}^{*}-\|\cdot\|_{G}}{\longrightarrow} x$.

Theorem 3.6. Let $\mathcal{I}$ be an admissible ideal in $\mathbb{N}$ and $\left(x_{k}\right)$ be a sequence in the $\operatorname{GNLS}\left(X,\|\cdot\|_{G}\right)$ such that $x_{k} \stackrel{\mathcal{I}^{*}-\|\cdot\|_{G}}{\longrightarrow} x$. Then $x_{k} \stackrel{\mathcal{I}-\|\cdot\|_{G}}{\longrightarrow} x$.

Proof. Let us assume that $x_{k} \stackrel{\mathcal{I}^{*}-\|\cdot\|_{G}}{\longrightarrow} x$. Then, there exists $M=\left\{m_{1}<m_{2}<\right.$ $\left.\ldots<m_{k}<\ldots\right\} \in \mathcal{F}(\mathcal{I})$ such that for every $\varepsilon>0$ and $\xi \in(0,1]$, there exists $N\left(=N_{\varepsilon}(\xi)\right) \in \mathbb{N}$ such that $A_{\left\|x_{m_{k}}-x\right\|_{G}}(\xi)<\varepsilon \forall k>N$. Since $\mathcal{I}$ is admissible, we must have $C(\xi, \varepsilon)=\left\{k \in \mathbb{N}: A_{\left\|x_{k}-x\right\|_{G}}(\xi) \geq \varepsilon\right\} \subseteq(\mathbb{N} \backslash M) \cup\left\{m_{1}, m_{2}, \ldots, m_{N}\right\} \in \mathcal{I}$. Hence $x_{k} \stackrel{\mathcal{I}-\|\cdot\|_{G}}{\longrightarrow} x$.

Remark 3.2. Converse of the above theorem is not true in general. Consider Example 3.1. It was shown that $x_{k} \stackrel{\mathcal{I}-\|\cdot\|_{G}}{\longrightarrow} \mathbf{0}$. But the same sequence is not gradually $\mathcal{I}^{*}$-convergent to $\mathbf{0}$. Beacuse for any $H \in \mathcal{I}$ there exists $p \in \mathbb{N}$ such that $H \subseteq \bigcup_{j=1}^{p} D_{j}$ and as a consequence $D_{p+1} \subseteq \mathbb{N} \backslash H$. Let $M$ denote the set $\mathbb{N} \backslash H$, then $M \in \mathcal{F}(\mathcal{I})$ and $\left(x_{m_{k}}\right)$ is gradual convergent to $\left(0,0, . ., 0, \frac{1}{p+1}\right)$, not to $\mathbf{0}$. Hence $x_{k}$ is not gradually $\mathcal{I}^{*}$-convergent to $\mathbf{0 .}$

Theorem 3.7. Let $\mathcal{I}$ be an admissible ideal in $\mathbb{N}$ which satisfies the condition $A P$ and $\left(x_{k}\right)$ be a sequence in the GNLS $\left(X,\|\cdot\|_{G}\right)$ such that $x_{k} \stackrel{\mathcal{I}-\|\cdot\|_{G}}{\longrightarrow} x$. Then $x_{k} \stackrel{\mathcal{I}^{*}-\|\cdot\|_{G}}{\longrightarrow} x$.

Proof. Let us assume that $x_{k} \stackrel{\mathcal{I}-\|\cdot\|_{G}}{\longrightarrow} x$. Then, for every $\xi \in(0,1]$ and $\eta>0$, the set $C(\xi, \eta)=\left\{k \in \mathbb{N}: A_{\left\|x_{k}-x\right\|_{G}}(\xi) \geq \eta\right\} \in \mathcal{I}$. This enables us to construct a countable family of mutually disjoint sets $\left\{C_{m}(\xi)\right\}_{m \in \mathbb{N}}$ in $\mathcal{I}$ by considering

$$
C_{1}(\xi)=\left\{k \in \mathbb{N}: A_{\left\|x_{k}-x\right\|_{G}}(\xi) \geq 1\right\}
$$

and

$C_{m}(\xi)=\left\{k \in \mathbb{N}: \frac{1}{m} \leq A_{\left\|x_{k}-x\right\|_{G}}(\xi)<\frac{1}{m-1}\right\}=C\left(\xi, \frac{1}{m}\right) \backslash C\left(\xi, \frac{1}{m-1}\right)$, for $m \geq 2$.

Now since $\mathcal{I}$ satisfies the condition AP, so for the above countable collection $\left\{C_{m}(\xi)\right\}_{m \in \mathbb{N}}$, there exists another countable family of subsets $\left\{B_{m}(\xi)\right\}_{m \in \mathbb{N}}$ of $\mathbb{N}$ satisfying 


$$
C_{j}(\xi) \triangle B_{j}(\xi) \text { is finite } \forall j \in \mathbb{N} \text { and } B(\xi)=\bigcup_{j=1}^{\infty} B_{j}(\xi) \in \mathcal{I} .
$$

Let $\varepsilon>0$ be arbitrary. By Archimedean property we can choose $m \in \mathbb{N}$ such that $\frac{1}{m+1}<\varepsilon$ and hence the following inclusion holds

$$
\left\{k \in \mathbb{N}: A_{\left\|x_{k}-x\right\|_{G}}(\xi) \geq \varepsilon\right\} \subseteq\left\{k \in \mathbb{N}: A_{\left\|x_{k}-x\right\|_{G}}(\xi) \geq \frac{1}{m+1}\right\}=\bigcup_{j=1}^{m+1} C_{j}(\xi) \in \mathcal{I} .
$$

Using (3.4) we can say that there exists an integer $k_{0} \in \mathbb{N}$, such that

$$
\bigcup_{j=1}^{m+1} B_{j}(\xi) \cap\left(k_{0}, \infty\right)=\bigcup_{j=1}^{m+1} C_{j}(\xi) \cap\left(k_{0}, \infty\right)
$$

Choose $k \in \mathbb{N} \backslash B(\xi) \in \mathcal{F}(\mathcal{I})$ such that $k>k_{0}$. Then we must have $k \notin \bigcup_{j=1}^{m+1} B_{j}(\xi)$ and hence $k \notin \bigcup_{j=1}^{m+1} C_{j}(\xi)$. Thus we have, $A_{\left\|x_{k}-x\right\|_{G}}(\xi)<\frac{1}{m+1}<\varepsilon$. Hence we have $x_{k} \stackrel{\mathcal{I}^{*}-\|\cdot\|_{G}}{\longrightarrow} x$.

Definition 3.3. Let $\left(x_{k}\right)$ be a sequence in the GNLS $\left(X,\|\cdot\|_{G}\right)$. Then $\left(x_{k}\right)$ is said to be gradually $\mathcal{I}$-Cauchy if for every $\varepsilon>0$ and $\xi \in(0,1]$, there exists a natural number $N\left(=N_{\varepsilon}(\xi)\right)$ such that the set $C(\xi, \varepsilon)=\left\{k \in \mathbb{N}: A_{\left\|x_{k}-x_{N}\right\|_{G}}(\xi) \geq \varepsilon\right\} \in \mathcal{I}$.

Theorem 3.8. Let $\left(X,\|\cdot\|_{G}\right)$ be a GNLS. Then every gradually $\mathcal{I}$-convergent sequence in $X$ is gradually $\mathcal{I}$-Cauchy sequence.

Proof. Let $\left(x_{k}\right)$ be a sequence in $X$ such that $x_{k} \stackrel{\mathcal{I - \| \cdot \| _ { G }}}{\longrightarrow} x$. Then, for every $\varepsilon>0$ and $\xi \in(0,1]$,

$$
C(\xi, \varepsilon) \in \mathcal{I}, \text { where } C(\xi, \varepsilon)=\left\{k \in \mathbb{N}: A_{\left\|x_{k}-x\right\|_{G}}(\xi) \geq \varepsilon\right\} .
$$

Clearly, $\mathbb{N} \backslash C(\xi, \varepsilon) \in \mathcal{F}(\mathcal{I})$ and therefore, is non-empty. Choose $N\left(=N_{\varepsilon}(\xi)\right) \in$ $\mathbb{N} \backslash C(\xi, \varepsilon)$. Then we have $A_{\left\|x_{k}-x_{N}\right\|_{G}}(\xi)<\varepsilon$.

Let $B(\xi, \varepsilon)=\left\{k \in \mathbb{N}: A_{\left\|x_{k}-x_{N}\right\|_{G}}(\xi) \geq 2 \varepsilon\right\}$. Now we prove that the following inclusion is true

$$
B(\xi, \varepsilon) \subseteq C(\xi, \varepsilon) .
$$

For if $p \in B(\xi, \varepsilon)$ we have

$$
2 \varepsilon \leq A_{\left\|x_{p}-x_{N}\right\|_{G}}(\xi) \leq A_{\left\|x_{p}-x\right\|_{G}}(\xi)+A_{\left\|x-x_{N}\right\|_{G}}(\xi)<A_{\left\|x_{p}-x\right\|_{G}}(\xi)+\varepsilon,
$$

which implies $p \in C(\xi, \varepsilon)$. Thus we conclude that $B(\xi, \varepsilon) \in \mathcal{I}$, which means $\left(x_{k}\right)$ is gradually $\mathcal{I}$-Cauchy sequence. 
Definition 3.4. Let $\left(x_{k}\right)$ be a sequence in the GNLS $\left(X,\|\cdot\|_{G}\right)$. Then $\left(x_{k}\right)$ is said to be gradually $\mathcal{I}^{*}$-Cauchy if there exists a set $M=\left\{m_{1}<m_{2}<\ldots<m_{k}<\right.$ $..\} \in \mathcal{F}(\mathcal{I})$ such that the subsequence $\left(x_{m_{k}}\right)$ is gradual Cauchy sequence.

Theorem 3.9. Let $\mathcal{I}$ be an admissible ideal in $\mathbb{N}$ and $\left(x_{k}\right)$ be a sequence in the $\operatorname{GNLS}\left(X,\|\cdot\|_{G}\right)$. If $\left(x_{k}\right)$ is gradually $\mathcal{I}^{*}-$ Cauchy then it is gradually $\mathcal{I}$-Cauchy.

Proof. Suppose $\left(x_{k}\right)$ is gradually $\mathcal{I}^{*}-$ Cauchy. Then, there exists a set $M=\left\{m_{1}<\right.$ $\left.m_{2}<\ldots<m_{k}<..\right\} \in \mathcal{F}(\mathcal{I})$ such that for every $\varepsilon>0$, there exists $i_{0}\left(=i_{0}(\xi, \varepsilon)\right) \in \mathbb{N}$ such that $A_{\left\|x_{m_{i}}-x_{m_{j}}\right\|_{G}}(\xi)<\varepsilon$ holds for any $i, j>i_{0}$. Let $N\left(=N_{\varepsilon}(\xi)\right)=m_{i_{0}+1}$. Then we have for any $\varepsilon>0$,

$$
C(\xi, \varepsilon)=\left\{k \in \mathbb{N}: A_{\left\|x_{k}-x_{N}\right\|_{G}}(\xi) \geq \varepsilon\right\} \subseteq(\mathbb{N} \backslash M) \cup\left\{m_{1}, m_{2}, . ., m_{i_{0}}\right\} \in \mathcal{I} .
$$

Hence $\left(x_{k}\right)$ is gradually $\mathcal{I}$-Cauchy.

Acknowledgment: The first author is greatful to University Grants Commission, India for their fellowships funding under UGC-JRF scheme during the preparation of this paper.

\section{RE F E R E N C E S}

1. F. Aiche and D. DuBois: Possibility and gradual number approaches to ranking methods for random fuzzy intervals. Commun. Comput. Inf. Sci. 299 (2012), 9-18.

2. S. Debnath and D. Rakshit: On I-statistical convergence. Iran. J. Math. Sci. Inform. 13(2) (2018), 101-109.

3. K. Demirci: I-limit superior and limit inferior. Math. Commun. 6 (2001), 165172.

4. K. Dems: On I-Cauchy sequences. Real Anal. Exchange 30(1) (2004-2005), $123-128$.

5. D. Dubois and H. Prade: Gradual elements in a fuzzy set. Soft Comput. 12 (2007), 165-175.

6. M. Ettefagh, F. Y. AzAri and S. Etemad: On some topological properties in gradual normed spaces. Facta Univ. Ser. Math. Inform. 35(3) (2020), 549-559.

7. M. Ettefagh, S. Etemad and F. Y. Azari: Some properties of sequences in gradual normed spaces. Asian-Eur. J. Math. 13(4) (2020), 2050085.

8. J. Fortin, D. Dubois and H. FArgier: Gradual numbers and their application to fuzzy interval analysis. IEEE Trans. Fuzzy Syst. 16(2) (2008), 388-402.

9. J. Gogola, M. Macaj and T. Visnyai: On $I_{c}^{(q)}$-convergence. Ann. Math. Inform. 38 (2011), 27-36.

10. P. Kostyrko, M. Macaj, T. Salat and M. Sleziak: I-convergence and extremal I-limit points. Math. Slovaca 55(4) (2005), 443-464.

11. P. Kostyrko, T. Salat and W. Wilczynski: I-convergence. Real Anal. Exch. 26(2) (2000-2001), 669-686. 
12. L. LiETARD and D. ROCACHER: Conditions with aggregates evaluated using gradual numbers. Control Cybernet. 38 (2009), 395-417.

13. M. Mursaleen and S. A. Mohiuddine: On ideal convergence in probabilistic normed spaces. Math. Slovaca 62(1) (2012), 49-62.

14. A. Nabiev, S. Pehlivan and M. Gurdal: On I-Cauchy sequences. Taiwanese J. Math. 11(2) (2007), 569-576.

15. I. SAdeqi and F. Y. Azari: Gradual normed linear space. Iran. J. Fuzzy Syst. 8(5) (2011), 131-139.

16. T. Salat, B. C. Tripathy and M. Ziman: On some properties of I-convergence. Ann. Math. Inform. 38 (2011), 27-36.

17. E. SAvas and P. DAs: A generalized statistical convergence via ideals. Appl. Math. Lett. 24(6) (2011), 826-830.

18. Е. A. Sтоск: Gradual numbers and fuzzy optimization. Ph. D. Thesis, University of Colorado Denver, Denver, America, 2010.

19. B. C. TRIPAThy and B. HAZARIKA: Paranorm I-convergent sequence spaces. Math. Slovaca 59(4) (2009), 485-494.

20. L. A. ZADEH: Fuzzy sets. Inf. Control 8 (1965), 338-353.

21. C. Zhоu: Gradual metric spaces. Appl. Math. Sci. 9(14) (2015), 689-701.

22. C. Zhou and J. Li: New fuzzy measure based on gradual numbers. Int. J. Math. Anal. (N.S.) 9 (2015), 101-110. 